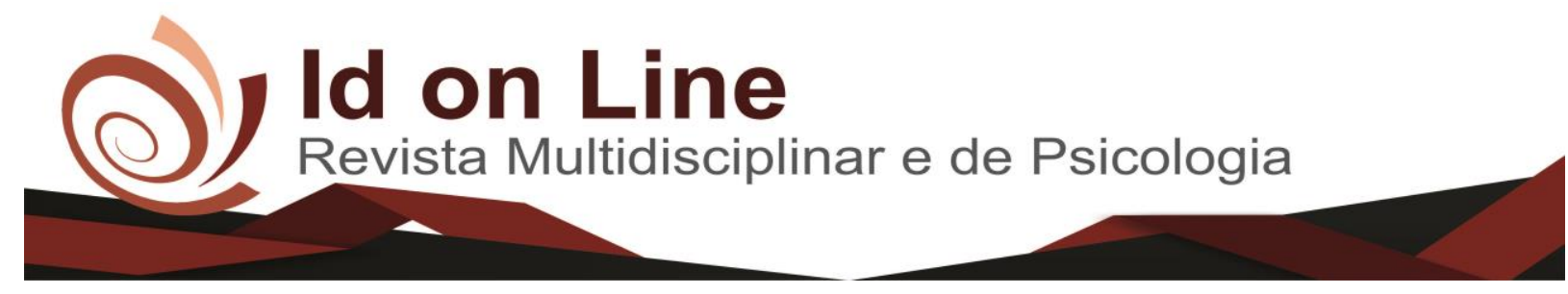

Artigo

\title{
Incontinência urinária e disfunção sexual em gestantes
}

\author{
Tamara Cordeiro Farias ${ }^{\prime}$; Karla Cavalcante Silva de Morais²; Rosana Porto Cirqueira3;
} Luciana Santos de Albuquerque ${ }^{4}$; Juliana Barros Ferreira ${ }^{5}$

Resumo: O objetivo deste estudo propõe analisar a associação entre incontinência urinária e disfunção sexual no período gestacional. Trata-se de uma pesquisa observacional, analítica e quantitativa. A amostra foi composta por 26 gestantes sexualmente ativas. As participantes responderam um questionário sociodemográfico e obstétrico, o International Consultation on Incontinence Questionnaire - Short Form e o Female Sexual Function Index. Foi realizada estatística descritiva e o teste de qui-quadrado de Pearson para a associação. Os resultados apontam que $53,8 \%$ das gestantes apresentaram incontinência urinária e 57,7\% apresentaram disfunção sexual. Não houve associação entre a variável incontinência urinária e função sexual ( $p>0,05)$. A gestação apresenta grandes repercussões funcionais, portanto medidas de prevenção e tratamento são necessárias para proporcionar uma assistência integral à saúde da mulher e uma melhor qualidade de vida durante a gestação.

Palavras-chave: Gravidez. Incontinência Urinária. Mulheres. Saúde Sexual. Sexualidade

\section{Urinary incontinence and sexual dysfunction in pregnant women}

\begin{abstract}
The aim of this study is to analyze the association between urinary incontinence and sexual dysfunction during the pregnancy period. This is an observational, analytical and quantitative research. The sample was composed by 26 pregnant women sexually active. The participants answered a socio-demographic and obstetric questionnaire, the International Consultation on Incontinence Questionnaire - Short Form and the Female Sexual Function Index. Descriptive statistics and Pearson chi-squared test were used for association. The results showed that $53,8 \%$ of the pregnant women presented urinary incontinence and $57,7 \%$ presented sexual dysfunction. There was no association between the rinary incontinence and sexual dysfunction variable $(\mathrm{p}>0,05)$. The gestation presents big functional repercussions, therefore preventive and treatment measures are needed to provide an integral attention to the woman's health and a better life quality during the pregnancy.
\end{abstract}

Keywords: Pregnancy. Urinary incontinence. Women. Sexual health. Sexuality

\footnotetext{
${ }^{1}$ Discente graduanda do curso de Fisioterapia pela Faculdade Independente do Nordeste-FAINOR. http://www.fainor.com.br, Vitória da Conquista - BA, Brasil.

${ }^{2}$ Fisioterapeuta. Docente da FAINOR e Maurício de Nassau. Mestre em Saúde Pública-ENSP/FIOCRUZ. Vitória da Conquista-BA, Brasil.

${ }^{3}$ Fisioterapeuta. Docente da FTC e FAINOR. Mestranda em Saúde Coletiva- UFBA/Ba, Brasil

${ }^{4}$ Fisioterapeuta. Docente da FAINOR. Especialista em Pilates: Atenção Múltipla a Saúde- Faculdade Guanambi/BA.

${ }^{5}$ Fisioterapeuta, Docente da FAINOR e FTC em Vitória da Conquista/BA. Mestre em Tecnologias em Saúde pela Escola Bahiana de Medicina e Saúde Pública, Salvador/Ba. Pesquisadora do Centro de Atenção ao Assoalho Pélvico- CAAP em Salvador - BA, Brasil.
}

*Autor Correspondente: Juliana Barros Ferreira, Rua Sifredo Pedral Sampaio, 414; Bairro Recreio; CEP: 45020-190; Vitória da Conquista/BA; julibarros78@ hotmail.com (77)98819 - 2774. 


\section{Introdução}

A Sociedade Internacional de Continência define incontinência urinária (IU) como toda perda involuntária de urina (ABRAMS et al., 2003). Esta incontinência pode ser classificada conforme os sintomas apresentados em três principais tipos: incontinência urinária de esforço, urgeincontinência e incontinência urinária mista (DIAS; RODRIGUES, 2016). Essa condição possui uma alta prevalência principalmente no público feminino, constituindo assim, um problema de saúde pública, pois, suas repercussões podem atingir negativamente a qualidade de vida dessas mulheres, seja pelo impacto físico, emocional, social e sexual (SACOMORI et al., 2013).

A IU apresenta uma taxa de prevalência no sexo feminino de 27,6 \% e na gestação esses valores podem variar entre 20\% e 67\% (MAGAJEWSKI; BECKHAUSER; GROTT, 2013). Nesse contexto, é válido destacar que a etiologia da IU é multifatorial, porém, a gravidez, devido às modificações mecânicas que ocorrem no assoalho pélvico e as mudanças hormonais, é considerada fator de risco ou um agravante importante (BELEZA, 2013).

Além dos problemas higiênicos e sociais, os sintomas urinários característicos do período gestacional podem ter influência na função sexual das gestantes. Pois, muitas mulheres relatam sentir-se incomodadas com a perda urinária, o que as deixam constrangidas durante a relação sexual, podendo desse modo, causar algum tipo de disfunção sexual (BELEZA, 2013).

A disfunção sexual (DS) é contextualizada como a falta, excesso ou qualquer alteração nas fases de desejo, excitação, orgasmo e resolução da relação sexual. (MATHIAS et al., 2015). O período gestacional, pelas alterações hormonais e funcionais pode causar um aumento significativo da DS (MACÊDO, 2017).

Portanto, a DS e a IU não devem ser negligenciadas pelos profissionais da área da saúde, visto que, a saúde sexual é importante para a durabilidade das relações afetivas, bem como faz parte da saúde integral e do bem-estar físico e social do ser humano (PRADO; LIMA; DE LIMA, 2013).

Atualmente, ainda é inexpressiva a investigação científica acerca da incontinência urinária e suas repercussões, principalmente as disfunções sexuais durante o ciclo gravídico. Nesse aspecto, este estudo se justifica pela necessidade de novas pesquisas, para dispor de dados epidemiológicos mais objetivos, a fim de melhorar o conhecimento das gestantes e dos profissionais de saúde em relação ao tema, objetivando a implementação de intervenções de 
prevenção, diagnóstico e tratamento, favorecendo uma assistência integral a saúde da mulher e uma melhor qualidade de vida durante a gestação. Para tanto, o presente estudo propõe analisar a relação entre incontinência urinária e disfunção sexual na gestação.

\section{Metodologia}

Trata-se de um estudo observacional, analítico, de corte transversal, com abordagem quantitativa. Os dados foram coletados entre os meses de julho e agosto de 2017, através de uma amostra não probabilística por conveniência, compreendida por 26 gestantes, usuárias do Sistema Único de Saúde (SUS), cadastradas e assistidas por um programa de pré-natal de uma Unidade Básica de Saúde (UBS) do município de Vitória da Conquista- BA. Foram adotados como critérios de inclusão gestantes maiores de 18 anos de idade, sexualmente ativas que estavam no segundo ou terceiro trimestre gestacional. Sendo excluídas as de alto risco e as que utilizavam medicações que interferem na função do trato urinário inferior.

As informações foram obtidas por meio da aplicação de um questionário sociodemográfico e obstétrico elaborado pelas pesquisadoras com a finalidade de levantar dados, tais como: idade, raça, estado civil, escolaridade, nível econômico, número de filhos e tipo de gestação. Posteriormente foram aplicados dois questionários: O International Consultation on Incontinence Questionnaire - Short Form (ICIQ-SF) e o Female Sexual Function Index (FSFI).

O ICIQ-SF é um questionário validado para o Brasil composto por quatro questões, que avaliam a frequência, gravidade e o impacto da incontinência urinária, além de oito itens de múltipla escolha relacionados às causas ou situações que ocorrem (TAMANINI et al., 2004). O escore geral varia entre 0 e 21 pontos, sendo obtido pela soma dos resultados das questões 3,4 e 5 . A incontinência urinária é detectada através de um escore $\geq 3$, partindo do pressuposto da definição de incontinência urinária, como qualquer queixa de perda involuntária de urina, considerando nesse caso incontinentes as mulheres que responderem "Apresentar perda urinária" no ICIQ-SF (DE ALMEIDA; MACHADO, 2012).

Já o FSFI segundo Pechorro et al. (2009) contêm 19 questões que avaliam a função sexual nas últimas quatro semanas à aplicação do questionário, apresentando escores em cada domínio. Para cada questão existe um padrão de resposta, as opções variam entre 0 e 5 de forma 
crescente em relação à presença da função questionada. Apenas nas questões sobre dor a pontuação é definida de forma invertida. Se o escore de algum domínio for igual à zero, isso significa que não foi referida pela entrevistada relação sexual nas últimas quatro semanas. Ao final é apresentado um escore total, resultado da soma dos escores de cada domínio multiplicada por um fator que homogeneíza a influência de cada domínio no escore final.

A análise é realizada reunindo as respostas em seis domínios diferentes: Desejo itens 1 e 2 do questionário x (0,6); Excitação itens 3, 4, 5 e 6 x (0,3); Lubrificação itens 7, 8, 9, e 10 x $(0,3)$; Orgasmo itens 11, 12 e 13x (0,4); Satisfação itens 14, 15 e 16 x $(0,4)$ e Dor itens 17, 18 e 19x $(0,4)$. O escore total varia de 2 a 36 pontos, se ele for menor ou igual a 26 designa disfunção sexual (PECHORRO et al., 2009).

A coleta de dados ocorreu em uma sala reservada e tranquila, para manter a privacidade das participantes e reduzir o risco de constrangimento. As gestantes foram informadas quanto aos objetivos da pesquisa, bem como seus riscos e benefícios. Ao aceitarem participar, assinaram o Termo de Consentimento Livre e Esclarecido (TCLE) respeitando os princípios éticos estabelecidos pela resolução 466/12 do Conselho Nacional de Saúde (CNS). Este estudo foi aprovado pelo Comitê de Ética e Pesquisa da Faculdade Independente do Nordeste sob o número de protocolo CAAE: 68229417.9.0000.5578 e Parecer: 2.116.916.

A tabulação e análise estatística descritiva foram realizadas através do Software Statiscal Package for the Social Sciences (SPSS) versão 22.0 for Windows. Para as correlações entre as variáveis do estudo, foi utilizado o teste de qui-quadrado de Pearson com uma significância de 0,05 .

\section{Resultados}

A amostra foi composta por 26 participantes com idade entre 18 a 24 anos. As características clínicas e sociodemográficas são mostradas na tabela 1. São predominantes as mulheres com gravidez de feto único e gestantes que não possuem outros filhos. 
Tabela 1. Dados sociodemográficos e obstétricos. Vitória da Conquista-BA, 2017.

\begin{tabular}{|c|c|c|c|}
\hline Variáveis & $\%$ respostas & $\mathbf{n}$ & $\%$ \\
\hline Faixa Etária & 100 & & \\
\hline $18-24$ anos & & 12 & 46,2 \\
\hline $25-30$ anos & & 8 & 30,8 \\
\hline $31-36$ anos & & 6 & 23,0 \\
\hline Raça & 100 & & \\
\hline Branca & & 5 & 19,2 \\
\hline Parda & & 17 & 65,4 \\
\hline Negra & & 4 & 15,4 \\
\hline Estado civil & 100 & & \\
\hline Solteira & & 4 & 15,4 \\
\hline Casada & & 16 & 61,5 \\
\hline União Estável & & 6 & 23,1 \\
\hline Escolaridade & 100 & & \\
\hline Ensino fundamental incompleto & & 7 & 26,9 \\
\hline Ensino fundamental completo & & 2 & 7,7 \\
\hline Ensino médio incompleto & & 4 & 15,4 \\
\hline Ensino médio completo & & 11 & 42,3 \\
\hline Ensino superior & & 2 & 7,7 \\
\hline Renda familiar & 100 & & \\
\hline Sem rendimento & & 3 & 11,54 \\
\hline 1 salário mínimo & & 17 & 65,38 \\
\hline Até 2 salários mínimos & & 3 & 11,54 \\
\hline De 2 a 5 salários mínimos & & 3 & 11,54 \\
\hline Tipo de gestação & 100 & & \\
\hline Única & & 25 & 96,2 \\
\hline Não sabe & & 1 & 3,8 \\
\hline Possui outros fillhos & 100 & & \\
\hline Sim & & 12 & 46,2 \\
\hline Não & & 14 & 53,8 \\
\hline
\end{tabular}

Fonte: Dados da pesquisa

A tabela 2 indica dados referentes à perda urinária e impacto na vida diária. Dentre as gestantes entrevistadas 53,8\% são incontinentes (ICIQ-SF $\geq 3$ ). Em relação ao impacto da IU, $23,1 \%$ indicaram moderada interferência na vida diária. $23 \%$ das participantes relataram perder urina ao tossir e espirrar. 
Tabela 2. Dados referentes à perda urinária e impacto na vida diária pelo ICIQ-SF das 26 gestantes. Vitória da Conquista-BA, 2017.

\begin{tabular}{|c|c|c|c|}
\hline Variáveis & $\%$ respostas & $\mathbf{n}$ & $\%$ \\
\hline Presença de incontinência urinária & 100 & & \\
\hline Não & & 12 & 46,2 \\
\hline Sim & & 14 & 53,8 \\
\hline Frequência & 100 & & \\
\hline Nunca & & 12 & 46,2 \\
\hline Uma vez por semana & & 7 & 26,9 \\
\hline Uma vez ao dia & & 1 & 3,8 \\
\hline Diversas vezes ao dia & & 6 & 23,1 \\
\hline Gravidade da IU & 100 & & \\
\hline Nenhuma & & 12 & 46,2 \\
\hline Pequena quantidade & & 11 & 42,3 \\
\hline Moderada quantidade & & 2 & 7,7 \\
\hline Grande quantidade & & 1 & 3,8 \\
\hline Impacto da IU & 100 & & \\
\hline Nada & & 14 & 53,84 \\
\hline Leve & & 3 & 11,5 \\
\hline Moderado & & 6 & 23,1 \\
\hline Grave & & 2 & 7,7 \\
\hline Muito grave & & 1 & 3,86 \\
\hline \multicolumn{4}{|l|}{ Situações ou causas de perda urinária } \\
\hline Nunca & & 12 & 46,1 \\
\hline Perco antes de chegar ao banheiro & & 5 & 19,2 \\
\hline Perco quando tusso ou espirro & & 6 & 23,0 \\
\hline $\begin{array}{l}\text { Perco quando estou fazendo atividades } \\
\text { físicas }\end{array}$ & & 3 & 11,5 \\
\hline $\begin{array}{l}\text { Perco quando terminei de urinar e estou me } \\
\text { vestindo }\end{array}$ & & 4 & 15,3 \\
\hline Perco sem razão óbvia & & 3 & 11,5 \\
\hline Perco o tempo todo & & 3 & 11,5 \\
\hline Perco quando estou dormindo & & 1 & 3,8 \\
\hline
\end{tabular}

Fonte: Dados da pesquisa

A prevalência da disfunção sexual nessa amostra corresponde a 57,7\%, com média de 23,16 \pm 2,92 do escore do FSFI. Os principais domínios afetados são: desejo, excitação e dor (Tabela 3). 
Tabela 3. Dados referente à função sexual das 26 gestantes avaliadas pelo FSFI. Vitória da Conquista-BA, 2017.

\begin{tabular}{lcc} 
Domínios & $\begin{array}{c}\text { Média } \pm \text { Desvio padrão } \\
(\mathrm{n}=15) \text { Disfunção }\end{array}$ & $\begin{array}{c}\text { Média } \pm \text { Desvio padrão } \\
(\mathrm{n}=11) \text { Ausência de disfunção }\end{array}$ \\
\hline Desejo & $2,92 \pm 0,84$ & $4,55 \pm 0,82$ \\
Excitação & $3,4 \pm 0,89$ & $4,71 \pm 0,69$ \\
Lubrificação & $4,2 \pm 0,91$ & $5,3 \pm 0,83$ \\
Orgasmo & $4,1 \pm 0,66$ & $4,98 \pm 1,13$ \\
Satisfação & $4,8 \pm 1,06$ & $5,52 \pm 0,46$ \\
Dor & $3,7 \pm 1,09$ & $5,12 \pm 0,92$ \\
Escore & $23,16 \pm 2,92$ & $30,22 \pm 3,03$ \\
\hline
\end{tabular}

Dados: Fontes da pesquisa

A associação entre incontinência urinária e disfunção sexual, não foi significativa ( $p$ > 0,05). Em contrapartida, quando avaliados alguns domínios do FSFI de forma isolada, houve correlação com a frequência, gravidade e impacto da IU ( $<$ 0,05) (Tabela 4).

Tabela 4. Associação entre função sexual e o International Consultation on Incontinence Questionnaire - Short Form. Vitória da Conquista - BA, 2017.

\begin{tabular}{lcccc}
\hline \multicolumn{1}{c}{ FSFI } & \multicolumn{3}{c}{ ICIQ-SF } \\
\cline { 2 - 5 } Desejo & 0,53 & 0,06 & $0,009^{*}$ & Presença de IU \\
Excitação & 0,43 & $0,04^{*}$ & $0,005^{*}$ & 0,52 \\
Lubrificação & 0,37 & 0,46 & 0,26 & 0,38 \\
Orgasmo & 0,46 & 0,30 & 0,33 & 0,30 \\
Satisfação & $0,01^{*}$ & $0,006^{*}$ & $0,002^{*}$ & 0,60 \\
Dor & 0,14 & 0,26 & 0,19 & 0,14 \\
Disfunção & 0,67 & 0,41 & 0,48 & 0,46 \\
\hline
\end{tabular}

*p $<0,05$ (significante pelo qui-quadrado de Person)

Fonte: Dados da pesquisa

\section{Discussão}

A gestação é um período marcado por inúmeras alterações físicas e fisiológicas no corpo feminino, por esse motivo, alterações importantes podem surgir principalmente no sistema geniturinário, desencadeando incontinência urinária e disfunções sexuais. 
Nesse estudo, a maior parte das gestantes eram jovens, não possuíam outros filhos e apresentaram perda urinária durante o período gestacional. De acordo com Mocellin, Rett e Driusso (2014), a idade e a paridade são fatores importantes para o desenvolvimento de sintomas urinários durante a gestação. Segundo estes autores, mulheres que possuem mais de 35 anos têm risco maior de desenvolverem disfunções do assoalho pélvico, em decorrência do envelhecimento fisiológico. Além disso, gestações anteriores podem aumentar a prevalência de sintomas miccionais. No estudo de Magajewski, Beckhauser e Grott (2013), realizado com 144 primigestas internadas em um hospital do Estado de Santa Catarina, observou-se que 58,7\% das participantes relataram no ICIQ-SF sintomas de IU. Dados bem menores do que o indicado por Oliveira et al. (2013) em uma pesquisa semelhante realizada com 495 mulheres, na qual $71 \%$ apresentaram IU nas últimas quatro semanas de gestação.

Além do mais, houve maior prevalência dos sintomas compatíveis com a IU aos esforços, corroborando assim, com os resultados de Sarcomori et al. (2013), em um estudo desenvolvido com 242 mulheres, no qual, a maioria das gestantes perdia uma pequena quantidade de urina e as situações e/ou causas em que ocorriam eram durante tosse ou espirro.

Baracho (2012) explica que durante o segundo e terceiro trimestre gestacional, a bexiga que antes era um órgão pélvico, passa a ser intra-abdominal, em consequência do aumento progressivo do útero e pela presença do polo cefálico, que a eleva para cima. O resultado dessa alteração é o aumento da pressão intra-abdominal e um ângulo uretrovesical modificado, contribuindo com o desejo miccional e a incontinência. Além disso, há um aumento significativo nas concentrações de diversos hormônios, dentre eles, estão à progesterona e a relaxina. A primeira está intimamente ligada ao relaxamento da musculatura lisa, e a relaxina é a responsável pela diminuição do tônus e força do assoalho pélvico, o que também contribui para a perda urinária durante a gestação (ARAGÃO et al., 2013).

Essa pesquisa, mostra que a IU causa moderado impacto na vida diária das participantes, e os resultados vão de encontro com o estudo de Mathias et al. (2014) realizado com gestantes acompanhadas em postos de saúde de duas cidades do nordeste brasileiro, no qual, a maioria referiu que a perda urinária interfere de leve a moderadamente na sua vida diária.

Segundo Henkes et al. (2015), a IU na mulher pode trazer além do comprometimento físico, alterações psicossociais, gerando limitação na autonomia e autoestima, comprometendo de forma importante a qualidade de vida. Este comprometimento, pode interferir nas interações 
sociais, atividades domésticas, ocupacionais, sexuais, além da auto percepção do estado geral de saúde, causando morbidades, estresse ou até mesmo debilidade.

A incontinência urinária, também pode impactar de forma significativa a função sexual das mulheres. Visto que, muitas têm medo de perder urina durante o ato sexual, ou de interromper a relação para urinar. Desse modo, a perda urinária pode tornar-se um obstáculo para o envolvimento e satisfação sexual, pois, os sentimentos negativos, podem levar a mulher a se sentir impotente por não conseguir controlar uma função do próprio corpo. Esta condição pode gerar muitas vezes, frustação, depressão e isolamento social (CRUZ, 2015).

No que diz respeito à Disfunção Sexual, Mathias et al. (2014) encontrou prevalência de $42,6 \%$ ao aplicar o FSFI em 54 gestantes durante o terceiro trimestre gestacional. Ao avaliar os domínios sexuais isoladamente, Prado, Lima e De Lima (2013) observaram que os domínios sexuais mais prejudicados são desejo e excitação, dados similares aos evidenciados no presente estudo.

Lima, Dotto e Mamede (2013) trazem que no primeiro trimestre de gestação 40\% das mulheres sofrem redução na frequência sexual. Já no segundo e terceiro trimestre, essa diminuição é de $30 \%$ e $60 \%$ respectivamente. Segundo Pereira (2015), o primeiro trimestre gestacional é um momento marcado por alterações hormonais, ocorrendo um aumento significativo na produção de progesterona e estrógeno e uma redução das gonadotrofinas. Isto resulta em elevação do volume plasmático, provocando assim, náuseas, sonolência, fadiga e hipersensibilidade nas mamas, o que desencadeia redução do desejo sexual.

No segundo trimestre, ocorre uma maximização do desejo sexual, em relação ao primeiro, pois, verifica-se uma estabilização hormonal e uma redução dos sintomas característicos do trimestre anterior, além de uma lubrificação mais eficaz, que favorece o desempenho sexual (PEREIRA, 2015). Bezerra et al. (2015) afirma que o último trimestre gestacional, é o período em que mais ocorre modificações físicas, e por esse motivo, pode haver uma redução significativa na libido e na atividade sexual, visto que, é uma fase marcada por aumento do peso fetal, volume abdominal, desconfortos, fadiga, dores, falta de ar, ansiedade pela proximidade do parto e consequentemente pouca atratividade pelo sexo.

A dor também foi um domínio afetado significativamente nessa amostra. Segundo Sperandio et al. (2016) a presença de dor ou algum desconforto durante a tentativa ou penetração vaginal completa, ocorre principalmente no terceiro trimestre gestacional, em função da tração inferior da musculatura do assoalho pélvico, causada pelo aumento do peso 
uterino que altera a contratilidade desses músculos e pelo aparecimento de veias varicosas na região vulvar e no interior da vagina.

Nesse estudo, não houve associação significativa entre a IU e DS. Bomfim e Melro (2014) trazem que a sexualidade do casal nessa nova etapa pode estar alterada, devido a certos preconceitos e tabus vivenciados pela mulher e seu parceiro. Isso inclui a falta de conhecimento em tornar a relação sexual mais prazerosa e o medo de prejudicar ou atingir o feto durante o exercício sexual.

O relacionamento com o parceiro também é algo que deve ser levado em consideração, pois o vínculo emocional nessa fase está fortemente associado à satisfação sexual. Geralmente, as gestantes que relatam maior grau de satisfação conjugal são aquelas que nunca tiveram filhos, as que planejaram sua gestação e têm um vinculo estável com o seu parceiro (PEREIRA, 2015). Desse modo, é importante destacar que a saúde sexual está intimamente ligada ao equilíbrio físico, emocional e social. Qualquer desordem nessas vertentes pode resultar em insatisfação sexual e contribuir para o desenvolvimento das DS (CARTEIRO; SOUSA; CALDEIRA, 2016).

A pesquisa em questão apresentou algumas limitações, inicialmente por ser um estudo transversal e pelo fato dos dados serem obtidos através de questionários com dados objetivos. Nesse aspecto, seria também importante incorporar uma abordagem qualitativa à pesquisa, durante todo o período gestacional e pós-parto.

\section{Considerações finais}

Com base no presente estudo, constatou-se que não houve associação significativa entre incontinência urinária e disfunção sexual na gestação. Em contrapartida, observa-se alta prevalência de sintomas urinários e sexuais nesse período, revelando um dado preocupante.

Mediante essas constatações, é preciso investigar e fortalecer dentro das políticas de saúde, a prioridade da mulher no ciclo gravídico-puerperal com o intuito de reduzir essa comorbidade causada nesse período.

Dessa forma, faz-se necessário uma atenção qualificada, a fim de promover ações de prevenção e tratamento. Portanto, a contribuição desse estudo é ampliar o conhecimento das gestantes, profissionais e gestores da saúde sobre esta temática, com o intuito de levantar dados mais precisos, proporcionando maior conhecimento epidemiológico e possíveis intervenções. 


\section{Referências}

ABRAMS, P. et al. The standardisation of terminology in lower urinary tract function: report from the standardisation sub-committee of the International Continence Society. Urology, v. 61, n. 1, p. 37-49, 2003.

ARAGÃO, A.S. et al. Prevalência de Incontinência Urinária de Esforço em Gestantes Adolescentes. CORPVS, v. 1, n. 27, p. p. 32-37, 2013.

BARACHO, E. Fisioterapia Aplicada à Saúde da Mulher. Rio de Janeiro: Guanabara Koogan, 2012.

BELEZA, A.C.S. Sinais e sintomas de disfunção do trato urinário inferior em gestantes de um município do interior do estado de São Paulo. Fisioterapia Brasil, v. 14, n. 2, 2013.

BEZERRA, I.F.D. et al. Comparação da qualidade de vida em gestantes com disfunção sexual. Rev. bras. ginecol. obstet, v. 37, n. 6, p. 266-271, 2015.

BOMFIM, I.Q.M; MELRO, B.C.F. Estudo Comparativo da Função Sexual em Mulheres Durante o Período Gestacional. Journal of Health Sciences, v. 16, n. 4, 2015.

CARTEIRO, D.M.H; SOUSA, L.M.R. de; CALDEIRA, S.M.A. Indicadores clínicos de disfunção sexual em mulheres grávidas: revisão integrativa de literatura. Revista Brasileira de Enfermagem, v. 69, n. 1, p. 165-173, 2016.

CRUZ, M.L.S.C. et al. Implicações da incontinência urinária na sexualidade da mulher. 2015. Tese de Doutorado.

DE ALMEIDA, P.P.; MACHADO, L.R.G. A prevalência de incontinência urinária em mulheres praticantes de jump. Fisioterapia em Movimento, v. 25, n. 1, 2012.

DIAS, S.F.L.; RODRIGUES, A.M.S. A prevalência de incontinência urinária em mulheres nulíparas. J. Health Sci. Inst, v. 34, n. 1, p. 49-52, 2016.

HENKES, D.F. et al. Incontinência urinária: o impacto na vida de mulheres acometidas e o significado do tratamento fisioterapêutico. Semina: Ciências Biológicas e da Saúde, v. 36, n. 2, p. 45-56, 2016.

LIMA, A.C. de; DOTTO, L.M.G; MAMEDE, M.V. Prevalência de disfunção sexual em primigestas, no Município de Rio Branco, Acre, Brasil. Cadernos de Saúde Pública, v. 29, n. 8, p. 1544-1554, 2013.

MACÊDO, L.C. et al. Avaliação da função sexual em primíparas após parto vaginal e nuligestas. Revista Pesquisa em Fisioterapia, v. 7, n. 1, 2017. 
MAGAJEWSKI, F.R.L.; BECKHAUSER, M.T.; GROTT, Y. Prevalência de incontinência urinária em primigestas em um hospital no sul do Brasil. Arq Catarin Med, v. 42, n. 3, p. 54$58,2013$.

MATHIAS, A. E. R.A et al. Prevalência de incontinência urinária durante o terceiro trimestre Gestacional. Arq. Ciênc. Saúde. v. 21, n. 4, p.101-105, 2014.

MOCCELLIN, A.S.; RETT, M.T.; D, P. Incontinência urinária na gestação: implicações na qualidade de vida. Revista Brasileira de Saúde Materno Infantil, v. 14, n. 2, 2014.

OLIVEIRA, C. et al. Urinary incontinence in pregnant women and its relation with sociodemographic variables and quality of life. Revista da Associação Médica Brasileira (English Edition), v. 59, n. 5, p. 460-466, 2013.

PECHORRO, P. et al. Validação portuguesa do índice de Funcionamento Sexual Feminino (FSFI). Laboratório de Psicologia, v. 7, n. 1, p. 33-44, 2009.

PEREIRA, M. C. Avaliação da função sexual da mulher no período gestacional. 2015. 97 f. Dissertação (Mestrado em Enfermagem) - Universidade Federal de Alfenas, Alfenas, MG, 2015.

PRADO, D.S.; LIMA, R.V.; DE LIMA, L.M.M.R. Impacto da gestação na função sexual feminina. Rev. bras. ginecol. obstet, v. 35, n. 5, p. 205-209, 2013.

SACOMORI, C. et al. Prevalência e variáveis associadas à incontinência urinária no terceiro trimestre gestacional. Revista Brasileira de Saude Materno Infantil, v. 13, n. 3, 2013.

SPERANDIO, F.F. et al. Prevalência de dispareunia na gravidez e fatores associados. Revista Brasileira de Saude Materno Infantil, v. 16, n. 1, 2016.

TAMANINI, J.T.N. et al. Validation of the" international consultation on incontinence questionnaire-short form"(ICIQ-SF) for Portuguese. Revista de saude publica, v. 38, n. 3, p. 438-444, 2004.

\section{Como citar este artigo (Formato ABNT):}

FARIAS, Tamara C.; MORAIS, Karla C.S de; CIRQUEIRA, Rosana P.; ALBUQUERQUE, Luciana S. de; FERREIRA, Juliana B. Incontinência urinária e disfunção sexual em gestantes. Id on Line Revista Multidisciplinar e de Psicologia, 2017, vol.11, n.38, p. 237-248. ISSN: 1981-1179.

Recebido: 25.10 .2017

Aceito: 26.10 .2017 